\title{
24-hour accelerometry in COPD: Exploring physical activity, sedentary behavior, sleep and clinical characteristics
}

This article was published in the following Dove Medical Press journal: International Journal of COPD

\author{
Mark W Orme ${ }^{1-3}$ \\ Michael C Steiner ${ }^{1-3}$ \\ Mike D Morgan' \\ Andrew $\mathrm{P}$ Kingsnorth ${ }^{2,3}$ \\ Dale W Esliger ${ }^{2-4}$ \\ Sally J Singh ${ }^{1-3, *}$ \\ Lauren B Sherar ${ }^{2-4, *}$ \\ 'Centre for Exercise and \\ Rehabilitation Science, NIHR \\ Leicester Biomedical Research \\ Centre - Respiratory, Leicester, UK; \\ ${ }^{2} \mathrm{School}$ of Sport, Exercise and Health \\ Sciences, Loughborough University, \\ Loughborough, UK; ${ }^{3}$ National Centre \\ for Sport and Exercise Medicine, \\ Loughborough, UK; ${ }^{4}$ NIHR Leicester \\ Biomedical Research Centre, \\ Leicester, UK \\ *These authors contributed equally \\ to this work
}

Correspondence: Mark W Orme Centre for Exercise and Rehabilitation Science, NIHR Leicester Biomedical Research Centre - Respiratory, Glenfield Hospital, Groby Road, Leicester LE3 9QP, UK

Tel +44 II 62502762

Email mark.orme@uhl-tr.nhs.uk
Background: The constructs and interdependency of physical behaviors are not well described and the complexity of physical activity (PA) data analysis remains unexplored in COPD. This study examined the interrelationships of 24-hour physical behaviors and investigated their associations with participant characteristics for individuals with mild-moderate airflow obstruction and healthy control subjects.

Patients and methods: Vigorous PA (VPA), moderate-to-vigorous PA (MVPA), light PA (LPA), stationary time (ST), average movement intensity (vector magnitude counts per minute), and sleep duration for 109 individuals with COPD and 135 healthy controls were obtained by wrist-worn accelerometry. Principal components analysis (PCA) examined interrelationships of physical behaviors to identify distinct behavioral constructs. Using the PCA component loadings, linear regressions examined associations with participant (+, positive correlation; -, negative correlation), and were compared between COPD and healthy control groups.

Results: For both groups PCA revealed ST, LPA, and average movement intensity as distinct behavioral constructs to MVPA and VPA, labeled "low-intensity movement" and "high-intensity movement," respectively. Sleep was also found to be its own distinct behavioral construct. Results from linear regressions supported the identification of distinct behavioral constructs from PCA. In COPD, low-intensity movement was associated with limitations with mobility $(-)$, daily activities (-), health status (+), and body mass index (BMI) (-) independent of highintensity movement and sleep. High-intensity movement was associated with age $(-)$ and self-care limitations (-) independent of low-intensity movement and sleep. Sleep was associated with gender $(0=$ female, $1=$ male; $[-])$, lung function $(-)$, and percentage body fat $(+)$ independent of low-intensity and high-intensity movement.

Conclusion: Distinct behavioral constructs comprising the 24-hour day were identified as "lowintensity movement," "high-intensity movement," and "sleep" with each construct independently associated with different participant characteristics. Future research should determine whether modifying these behaviors improves health outcomes in COPD.

Keywords: accelerometry, COPD, physical activity, principal components analysis, sedentary behavior

\section{Introduction}

For over a decade, an abundance of research about the physical activity (PA) of individuals with COPD has been published. Compared to people with more severe disease, fewer studies have explored the PA of individuals with mild-moderate airflow obstruction. ${ }^{1}$ This is despite these patients taking fewer steps per day than healthy adults ${ }^{2}$ and experiencing significant respiratory impairment during activities of daily living. ${ }^{3}$ 
A lack of PA has been prospectively associated with greater risk of premature mortality, ${ }^{4}$ and increasing PA is recognized as an important goal of COPD management. ${ }^{5}$

In a review of PA methodologies in COPD, only $40 \%$ of studies were found to report intensity categories of PA, with most studies reporting daily step count. ${ }^{6}$ Daily step count does not describe the intensity at which movements are performed, an important aspect of PA for reducing the risk of hospitalization. ${ }^{7}$ In addition, as individuals with COPD are more sedentary than healthy adults ${ }^{8,9}$ an alternative approach to increasing PA by targeting reductions in sedentary behavior, defined as "any waking behavior characterized by an energy expenditure $\leq 1.5$ metabolic equivalents (METs), while in a sitting, reclining, or lying posture," ${ }^{10}$ has been put forward as a potentially more achievable target for patients. ${ }^{11}$ As time-based behaviors are inherently constrained by the 24-hour day, reducing time spent sedentary must result in spending more time in at least one other behavior. However, it is unclear whether this time would be replaced with light intensity activity, moderate-to-vigorous PA (MVPA), or sleep. With a pressing need to offer more personalized care, stratifying patients based on their physical behaviors may help to better tailor interventions to improve clinical outcomes. $^{12}$

Accelerometers allow the measurement of all physical behaviors: stationary time ([ST] a proxy measure of sedentary behavior), ${ }^{10}$ light activity, MVPA, and sleep. However, no study in COPD has explored how 24-hour behaviors relate to each other, how they are associated with demographics, social deprivation, clinical characteristics, and exercise capacity, and if these associations differ between behaviors and from healthy controls. Therefore, it remains unclear whether "not being sedentary" or "being physically active" are two sides of the same coin. This study aimed to examine the interrelationships of 24-hour physical behaviors and investigate their associations with participant characteristics in individuals with COPD with mild-moderate airflow obstruction and adults free from respiratory disease (controls).

\section{Patients and methods Study design}

Data was obtained from the PA and Respiratory Health (PhARaoH) Study; a cross-sectional, observational study of adults with and without a diagnosis of COPD according to primary care records. ${ }^{13}$ All participants provided written informed consent and the trial was conducted in accordance with the Declaration of Helsinki.

\section{Inclusion/exclusion criteria}

Participants were included if they were aged 40-75 years. The CONSORT flowchart for the present study is provided in Figure 1. Nonwhite British participants were excluded from the analysis due to a lack of sufficient sample size for individuals with COPD $(\mathrm{N}=5)$, with PA differences observed between ethnic groups ${ }^{14}$ impacting comparability between individuals with and without COPD. The final sample comprised of 109 individuals with COPD and 135 controls.

\section{Recruitment}

Individuals with COPD were recruited from general practice registers and control subjects were recruited through advertisements in community settings; both within Leicestershire, UK.

\section{Accelerometry}

Participants were asked to wear an ActiGraph wGT3X-BT accelerometer (ActiGraph, LLC, Pensacola, FL, USA) on their nondominant wrist for seven consecutive days, removed only for water-based activities. Valid day criteria of $\geq 10$ waking hours on $\geq 4$ days were used. ${ }^{15}$ Non-wear was defined as 90 minutes of consecutive zeros with allowance for 2 minutes of interruptions. ${ }^{16,17}$ Vector magnitude counts per minute (VMCPM) was used to denote average movement intensity. ST was defined as $<2,000 \mathrm{VMCPM}$, light PA (LPA) as 2,000-7,499 VMCPM, MVPA as $\geq 7,500$ VMCPM, and vigorous PA (VPA) as $\geq 8,250 \mathrm{VMCPM} .{ }^{18}$ Sleep duration was calculated using a sleep detection algorithm, which determined the onset and end of sleep using sustained periods

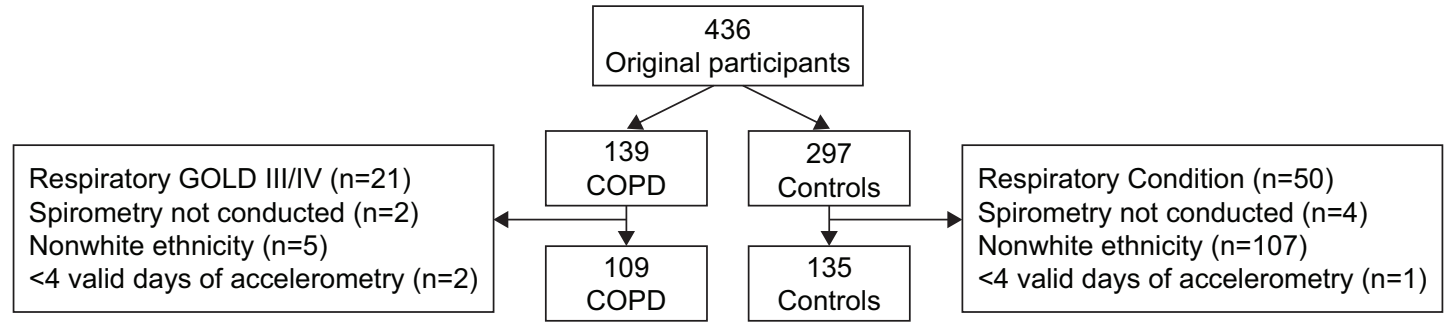

Figure I CONSORT flowchart.

Abbreviation: GOLD, Global Initiative for Obstructive Lung Disease. 
of (in)activity from VMCPM values based on the work of Carney et al. ${ }^{19}$ To identify the time when participants went to sleep, the algorithm identified consecutive dips in activity, specifically a $90 \%$ reduction from the previous epoch for 15 minutes between the hours of 21:00 and 23:59. To identify the time when sleep ended, the algorithm detected consecutive rises in activity level of at least $75 \%$ from the previous epoch for 5 minutes between 06:00 and 09:00. Further details of accelerometry methodology are provided as Supplementary Material. Patients were stratified by ST and MVPA to examine the proportion of patients considered "sedentary and not active," "sedentary and active," "not sedentary and not active," or "not sedentary and active" using pragmatic total per day thresholds of 600 minutes/day and 20 minutes/ day, respectively, similar to the mean values of the groups.

\section{Anthropometrics and body composition}

Height was measured using a portable stadiometer, and weight and percentage body fat were obtained using bioelectrical impedance analysis (Tanita MC780MA; Tanita Corporation, Tokyo, Japan) with BMI derived. Waist circumference was measured around the mid-point between the lowest rib and iliac crest. ${ }^{20}$

\section{Spirometry}

Lung function parameters were assessed using forced spirometry (MicroLab MK8 spirometer; CareFusion, San Diego, CA, USA) conducted in accordance with American Thoracic Society/European Respiratory Society guidelines, ${ }^{21}$ with the highest values for $\mathrm{FEV}_{1}$ and $\mathrm{FVC}$ used in the analyses. Severity of airflow obstruction was defined according to Global Initiative for Obstructive Lung Disease (GOLD) criteria $^{22}$ using established reference values. ${ }^{23}$

\section{Exercise capacity}

Exercise capacity was assessed using the incremental shuttle walk test (ISWT). ${ }^{24}$ The best distance from two tests was carried forward.

\section{Skeletal muscle strength}

Leg strength was assessed using the quadriceps maximal voluntary contraction (QMVC) test. ${ }^{25}$ Participants performed three sustained maximal isometric quadriceps contractions, with the greatest of the three efforts carried forward for analysis. Upper body skeletal muscle assessment was obtained by standing grip strength using a hand-held dynamometer (Takeii analog dynamometer; Takei Corp Ltd., Tokyo, Japan $)^{26}$ with the best result from three efforts carried forward.

\section{Questionnaires}

Self-reported breathlessness was obtained using the modified Medical Research Council (mMRC) dyspnea scale. ${ }^{27}$ The EuroQol EQ-5D-5L was used to assess perceived general health status comprising Likert scales for problems with mobility, self-care, usual activities, pain/discomfort, and anxiety and depression and a visual analog scale (0-100). ${ }^{28}$ Likert scales were used to form a general health index value. ${ }^{29}$ Participants self-reported their usual walking speed as "slow," "average," "fairly brisk," or "brisk."

\section{Statistical analyses}

Analyses were conducted using SPSS version 23.0 (IBM Corporation, Armonk, NY, USA) and all figures were produced using GraphPad Prism version 7 (GraphPad Software, Inc., La Jolla, CA, USA). Data are reported as mean (SD) or median (interquartile range [IQR]) with group comparisons performed using ANOVA or Mann-Whitney U test. Frequency comparisons between groups were performed using Chi-square test. Significance was set to $P<0.05$.

Times spent in physical behaviors were extrapolated to 1,440 minutes (24 hours) in order to standardize the wear time for each participant. PA (LPA, MVPA, VPA), ST, average movement intensity, and sleep duration were used in principal components analysis (PCA) to identify distinct behavioral components. These components were based on linear combinations of the encompassing variables weighted by their contribution to the explained variance within each respective component. PCA is robust against the multicollinearity present when dealing with time-based physical behaviors. The number of components produced by PCA and the component loadings (the correlation coefficients between the variables and components) are reported. Component scores were calculated for each participant for each component.

Univariate analyses were conducted using correlations between principal component scores and clinical characteristics. Linear regressions were used to examine variables associated with each principal component. All identified principal components were entered into the same regression models to determine associations with participant characteristics independent of other 24-hour behaviors. For all models, data were checked for linear relationship, absence of multicollinearity, homoscedasticity, and a normal distribution of residuals.

\section{Results \\ Sample characteristics}

Participant characteristics are provided in Table 1. Overall accelerometer adherence for 7 days was $94.7 \%$. Individuals with COPD spent more time stationary (616 \pm 111 vs $522 \pm 103$ minutes/ 
Table I Participant characteristics stratified by COPD status, reported as mean (SD) for continuous data

\begin{tabular}{|c|c|c|c|}
\hline & COPD $(n=109)$ & Controls $(n=135)$ & $P$-value \\
\hline \multicolumn{4}{|l|}{ Demographics } \\
\hline Age (years) & $65.7(7.1)$ & $58.5(9.0)$ & $<0.001$ \\
\hline Gender: female/male & $42 / 67$ & $89 / 46$ & $<0.001$ \\
\hline Employment status: employed/unemployed/retired & $28 / 12 / 69$ & $72 / 7 / 56$ & $<0.001$ \\
\hline $\begin{array}{l}\text { Household income }{ }^{\mathrm{a}}(£):<\in|8,000 / €| 8,000-30,999 / € 31,000-51,999 / \\
£ 52,000-99,999 / \geq £ \mid 00,000\end{array}$ & $40 / 31 / 17 / 6 / 0$ & $15 / 39 / 37 / 34 / 4$ & $<0.001$ \\
\hline \multicolumn{4}{|l|}{ Respiratory health } \\
\hline Smoking status: current/former/never & $18 / 78 / 12$ & $8 / 60 / 67$ & $<0.001$ \\
\hline Pack years (median [IQR]) & $38.9(29.0)$ & $10.6(18.0)$ & $<0.001$ \\
\hline $\mathrm{FEV}_{1}(\mathrm{~L})$ & $2.0(0.7)$ & $2.8(0.6)$ & $<0.001$ \\
\hline FEV $\%$ pred & $76.2(17.8)$ & $107.0(17.9)$ & $<0.001$ \\
\hline FVC (L) & $3.6(1.0)$ & $3.9(0.8)$ & 0.016 \\
\hline $\mathrm{FEV}_{1} / \mathrm{FVC}$ & $56.0(11.2)$ & $72.8(6.3)$ & $<0.001$ \\
\hline mMRC score (median [IQR]) & $I(I)$ & $0(1)$ & $<0.001$ \\
\hline \multicolumn{4}{|l|}{ Perceived general health } \\
\hline General health index value & $0.775(0.181)$ & $0.893(0.109)$ & $<0.001$ \\
\hline General health VAS score (median [IQR]) & $80(25)$ & $90(15)$ & $<0.001$ \\
\hline \multicolumn{4}{|l|}{ Body composition } \\
\hline BMI $\left(\mathrm{kg} / \mathrm{m}^{2}\right)$ & $28.6(5.6)$ & $26.9(5.2)$ & 0.016 \\
\hline Percentage body fat & $28.8(8.6)$ & $29.7(8.2)$ & 0.426 \\
\hline Waist circumference $(\mathrm{cm})$ & $99.9(13.9)$ & $89.9(14.4)$ & $<0.001$ \\
\hline \multicolumn{4}{|l|}{ Physical function } \\
\hline ISWT (m) & $387.4(158.5)$ & $580.1(189.5)$ & $<0.001$ \\
\hline QMVC (kg) & $34.9(13.2)$ & $38.3(14.7)$ & 0.067 \\
\hline Grip strength (kg) & $35.7(10.9)$ & $36.0(10.5)$ & 0.820 \\
\hline Usual walking speed: slow/average/fairly brisk/brisk & $29 / 63 / 15 / 2$ & $3 / 73 / 56 / 3$ & $<0.001$ \\
\hline
\end{tabular}

Note: ${ }^{\mathrm{I}} 5$ (13.7\%) COPD patients and six (4.5\%) controls did not provide an answer.

Abbreviations: BMI, body mass index; FEV \%pred, FEV, percentage predicted; ISWT, incremental shuttle walk test; IQR, interquartile range; mMRC, modified Medical Research Council; QMVC, quadriceps maximal voluntary contraction; VAS, visual analog scale.

day, $P<0.001)$ and accumulated less LPA $(369 \pm 99$ vs $405 \pm 79$ minutes/day, $P<0.001)$, MVPA (18 \pm 17 vs $38 \pm 30$ minutes/day, $P<0.001$ ), VPA (10 \pm 11 vs $23 \pm 21$ minutes/day, $P<0.001$ ), and sleep (438 \pm 88 vs $475 \pm 94$ minutes/day, $P=0.021$ ) and had a lower average movement intensity $(1,974 \pm 489$ vs $2,465 \pm 543$ VMCPM, $P<0.001)$ compared to controls.

\section{Associations between behaviors}

PCA classified ST and LPA as distinct components to MVPA and sleep for patients and controls revealing three distinct constructs: "low-intensity movement" (ST, LPA, and average movement intensity), "high-intensity movement" (MVPA and VPA), and "sleep" (sleep duration) (Table 2).

Table 2 PCA for sleep duration, VMCPM, sedentary time, light intensity PA, MVPA, and VPA (99.5\% of variance explained for COPD and $99.6 \%$ of variance explained for controls, $P<0.00 \mathrm{I}$ )

\begin{tabular}{|c|c|c|c|c|c|c|c|c|}
\hline \multirow{4}{*}{$\begin{array}{l}\text { Accelerometry } \\
\text { variables }\end{array}$} & \multicolumn{4}{|l|}{ COPD } & \multicolumn{4}{|l|}{ Controls } \\
\hline & \multirow{3}{*}{$\begin{array}{l}\text { Percent of } \\
\text { variance }\end{array}$} & \multicolumn{3}{|c|}{ Component loading } & \multirow{3}{*}{$\begin{array}{l}\text { Percent of } \\
\text { variance }\end{array}$} & \multicolumn{3}{|c|}{ Component loading } \\
\hline & & \multicolumn{3}{|c|}{ Component } & & \multicolumn{3}{|c|}{ Component } \\
\hline & & I & 2 & 3 & & 1 & 2 & 3 \\
\hline VMCPM & 54.8 & 0.897 & 0.428 & 0.010 & 18.2 & 0.679 & 0.714 & 0.135 \\
\hline Stationary & & -0.849 & -0.113 & -0.513 & & -0.410 & -0.839 & -0.355 \\
\hline LPA & & 0.953 & 0.098 & -0.282 & & 0.108 & 0.982 & -0.148 \\
\hline MVPA & 27.2 & 0.242 & $0.96 \mathrm{I}$ & -0.109 & 63.8 & 0.969 & 0.237 & 0.039 \\
\hline VPA & & 0.138 & 0.983 & -0.076 & & 0.979 & 0.184 & 0.039 \\
\hline Sleep & 17.5 & -0.039 & -0.151 & 0.998 & 17.5 & 0.042 & 0.030 & 0.999 \\
\hline
\end{tabular}

Notes: COPD: component I, “low-intensity movement”; component 2, "high-intensity movement”; and component 3, "sleep." Controls; component I, “high-intensity movement"; component 2, "low-intensity movement"; and component 3, "sleep." Bold values indicate the main contributing variables to each component.

Abbreviations: MVPA, moderate-to-vigorous PA; PA, physical activity; VMCPM, vector magnitude counts per minute (average movement intensity); VPA, vigorous PA; LPA, light PA. 
Within component one for COPD and component two for controls, ST was highly inversely related to LPA and average movement intensity.

Participants were stratified by their time in "low-intensity movement" and "high-intensity movement" using a ST threshold of $</ \geq 600$ minutes/day and MVPA threshold of $</ \geq 20$ minutes/day. For individuals with COPD (vs controls) $41 \%$ (vs 16\%) were "sedentary and not active," $12 \%$ (vs $7 \%$ ) were "sedentary and active," 24\% (vs 17\%) were "not sedentary and not active," and 23\% (vs 60\%) were "not sedentary and active" (Figure 2). These observations were confirmed when plotting the low-intensity and high-intensity movement component loadings (Figure S1). Association analyses presented from this point used component values for "low-intensity movement," "high-intensity movement," and "sleep."

\section{Univariate associations between physical behaviors and participant characteristics}

A schematic of the characteristics associated with the identified components is provided in Figure 3 with the strengths of associations ranging from very weak to moderate. For people with COPD "low-intensity movement," but not "highintensity movement," was associated with dyspnea, mobility limitations, activity limitations, general health index, BMI, and waist circumference (Table 3). "High-intensity movement," but not "low-intensity movement," was associated with age and self-care limitations. "Sleep", but not "lowintensity movement" or "high-intensity movement," was associated with gender, $\mathrm{FEV}_{1}$, and percentage body fat.
For healthy controls "low-intensity movement," but not "high-intensity movement," was associated with dyspnea and all body composition measures. "High-intensity movement," but not "low-intensity movement," was associated with age, self-reported pain, and usual walking speed. "Sleep", but not "low-intensity movement" or "high-intensity movement," was associated with gender and general health index.

\section{Independent associations between physical behaviors and participant characteristics}

All components were entered into a single model to examine the independent associations of physical behaviors with participant characteristics (eg, the association between high-intensity movement and age after controlling for lowintensity movement and sleep). In COPD, "low-intensity movement" was associated with limitations with mobility and daily activities, health status, BMI, and waist circumference independent of "high-intensity movement" and "sleep". "High-intensity movement" was associated with age and selfcare limitations independent of "low-intensity movement" and "sleep". "Sleep" was associated with gender, lung function, and percentage body fat independent of "low-intensity movement" and "high-intensity movement." For controls, "low-intensity movement" was associated with mMRC and body composition measures independent of "highintensity movement" and "sleep". "Sleep" was associated with gender independent of "low-intensity movement" and "high-intensity movement."

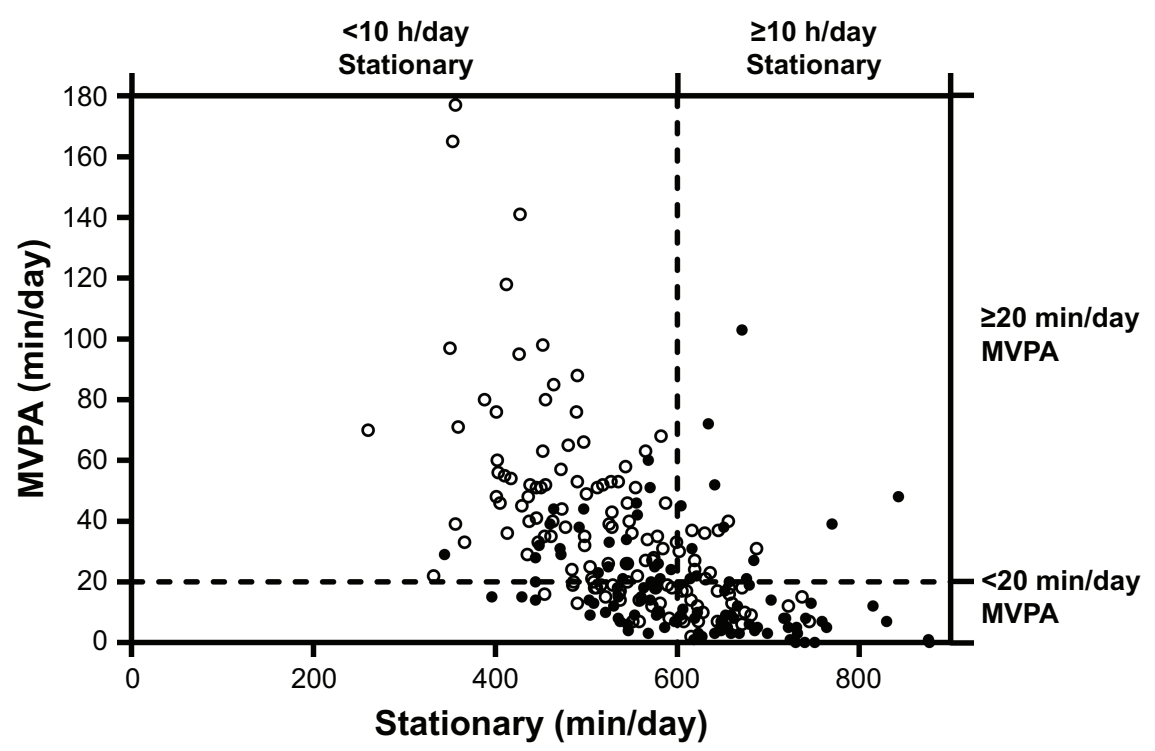

Figure 2 Scatterplot of sedentary time against MVPA (minutes/day) for individuals with COPD, stratified by their ST ( $</ \geq 600$ minutes/day) and MVPA ( $</ \geq 20$ minutes/day). Notes: Solid circles: COPD; blank circles: controls.

Abbreviations: MVPA, moderate-to-vigorous PA; PA, physical activity; ST, stationary time. 


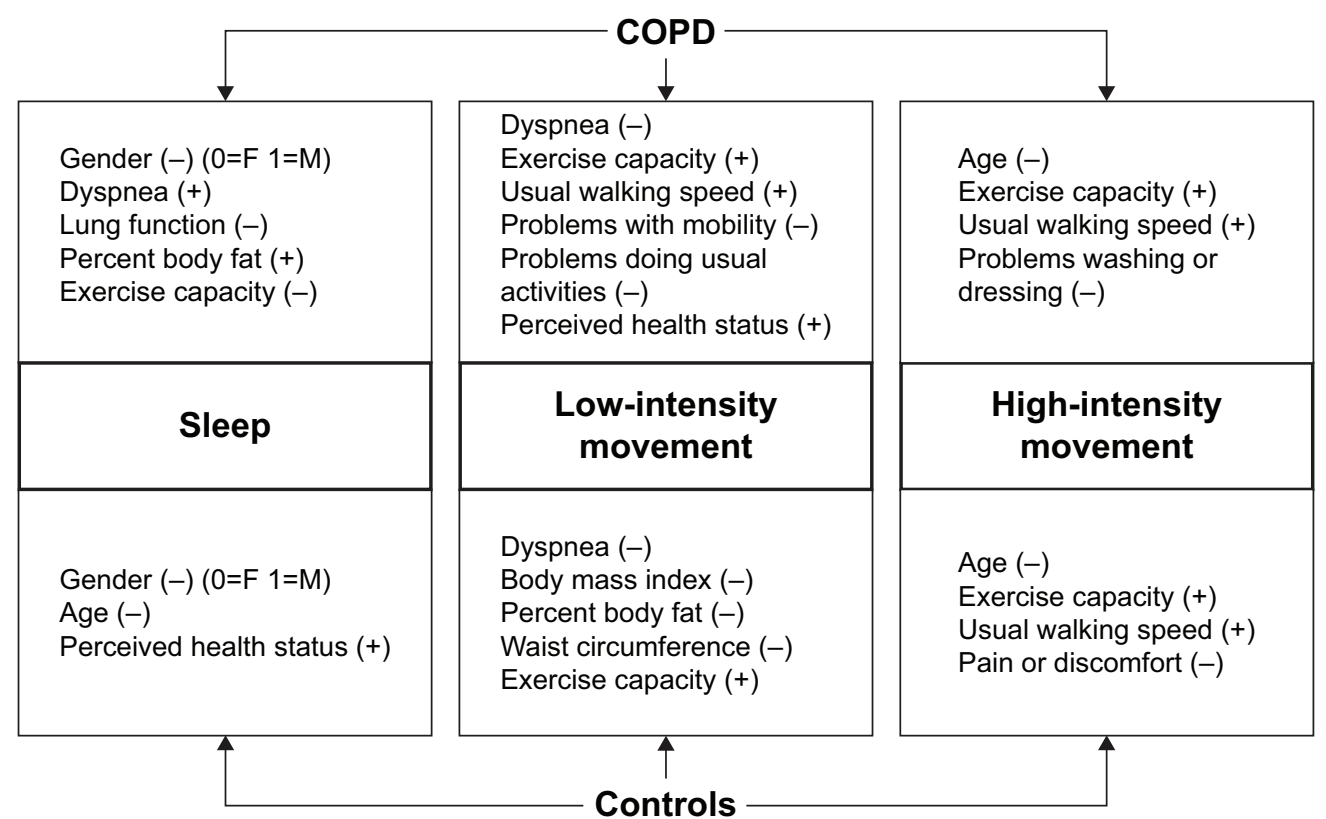

Figure 3 A schematic comparing the characteristics associated with sleep, low-intensity movement, and high-intensity movement for patients and controls. Notes: +, positive correlation between variables; -, negative correlation between variables.

Abbreviations: $\mathrm{F}$, female; $\mathrm{M}$, male.

\section{Discussion}

In the first study to explore the interrelationships of 24-hour physical behaviors in COPD, ST, light activity, and average movement intensity (construct labeled "low-intensity movement") were classified as distinct behavioral components to MVPA and VPA (construct labeled "high-intensity movement") as well as sleep duration. Therefore, being sedentary should not be interpreted as the opposite of being physically active for individuals with COPD. This was demonstrated by $12 \%$ of patients classified as "sedentary and active" and $24 \%$ of patients classified as "not sedentary and not active." Moreover, low-intensity and high-intensity movements were associated with different participant characteristics. Being stationary and doing light-intensity activity were highly proportional, classified within the same behavioral component, which should be accounted for using more sophisticated analyses in future studies.

Exploring 24-hour physical behaviors helps us understand how different interventions may influence patients' behaviors. Pulmonary rehabilitation promotes PA of a sufficient intensity (ie, MVPA) to evoke physical adaptations leading to improved cardiorespiratory fitness and muscle functioning ${ }^{30}$ and, therefore, does not specifically aim to reduce the time patients spend being stationary. Consequently, this type of intervention may not uniformly result in reductions in ST or changes in sleep duration. A study by Mesquita et $\mathrm{al}^{31}$ supports our finding that ST and light activity are highly proportional, with $86 \%$ of the patients completing pulmonary rehabilitation who reduced their ST also increasing their light intensity activity; with a strong correlation observed between changes in these behaviors $(r=-0.89) .{ }^{31}$ However, a strong correlation was also seen for changes in ST and changes in MVPA ( $r=-0.74$ ), with $82 \%$ of patients who reduced their ST also increasing their MVPA. ${ }^{31}$ A reason for this disparity may be the absence of a minimum threshold for change as most patients appeared to change their time spent in MVPA by \pm 5 minutes/day. ${ }^{31}$ Another potential reason and an important limitation of previous studies has been the use of conventional statistical analyses that are not sufficiently robust to handle perfectly multicollinear time-based behavioral variables. ${ }^{32}$

ST and taking part in light-intensity activity were found to be highly inversely proportional behaviors in the present study as indicated by the opposite signs for component loadings (eg, -0.849 for ST and 0.953 for light activity in COPD); unique from engaging in moderate and vigorous intensity activities. In support of our observation that ST and MVPA are distinct behaviors, a cross-sectional observational study of 1,001 individuals with COPD (all with severities of airflow obstruction) identified, using cluster analysis, patients who averaged $>1$ hour of MVPA per day based on the SenseWear armband whilst also accumulating $>11$ hours in behaviors classified as $<2.0$ METs. ${ }^{33}$ There is also a need to account for the 24-hour day rather than focusing only on waking behaviors. For example, patients with better sleep quality have been found to spend more time in light-intensity activity and MVPA the following day. ${ }^{34}$ Future work should explore the composition 


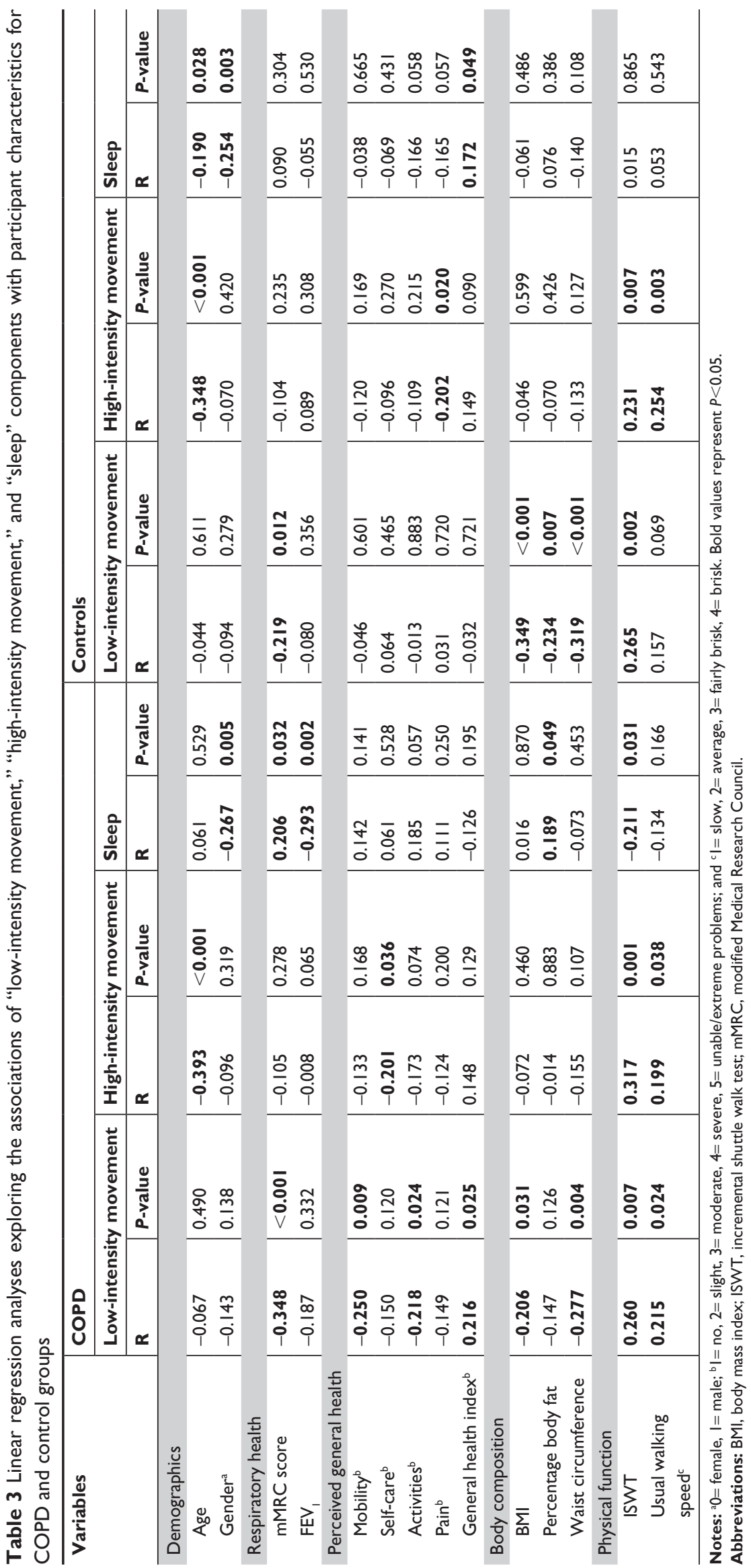


of the 24-hour day in relation to prospective health outcomes such as hospitalization and mortality using statistical methods that can account for the multicollinear nature of these data. ${ }^{32}$

Exercise therapy through pulmonary rehabilitation is effective in reducing the population symptom burden of COPD but its predication on high-intensity PA (MVPA) within a "one size fits all" framework may be improved through stratification approaches. By stratifying individuals by their MVPA and ST, the present study identified a particularly important subgroup of patients who may require exercise-based interventions to increase their MVPA in addition to behavioral approaches to reduce their ST (ie, help them become "not sedentary and active").

Pulmonary rehabilitation has been found to be equally beneficial to patients with mild disease compared to patients with more severe symptoms, ${ }^{35}$ yet these patients are markedly underrepresented in practice. ${ }^{36}$ Despite the observed associations between exercise capacity and all 24-hour physical behaviors, improvements in physical functioning and exercise capacity following pulmonary rehabilitation have inconsistently translated into subsequent increases in daily PA. ${ }^{37}$ Therefore, the weak to moderate strengths of associations observed in the present study are perhaps unsurprising. With the current evidence base primarily comprised of observational studies, future research is needed to determine whether interventions modifying physical behaviors can alter outcomes in COPD and whether patients with mild airflow obstruction may be more likely to increase their PA following pulmonary rehabilitation than those with more severe airflow obstruction.

Limitations of the present study must be considered, including the potential for recruitment bias and the cross-sectional design of the study. It is also unknown whether similar findings would be observed in patients with more severe airflow obstruction who are less likely to engage in as much MVPA as those with milder airflow obstruction. For example, for those patients unable to engage in MVPA, their time spent in PA and being sedentary would be equal opposites. The present sample was ethnically homogenous due to an insufficient number of nonwhite individuals with COPD $(\mathrm{N}=5)$, but this may represent the composition of ethnic groups living with COPD in Leicestershire. ${ }^{38}$ Groups were not matched for gender which may have confounded results. We were also unable to examine sleep quality in the present study, thus it should be noted that overall sleep duration may oversimplify the complexity of sleep and its potential relationship with waking behaviors. ${ }^{34}$ The use of wrist-worn accelerometry limited our ability to directly compare behaviors measured at more popular locations such as the waist or with more frequently used devices. Given the use of accelerometry to assess sedentariness, we were only able to assess ST rather than the specific postures required to classify sedentary behaviors. ${ }^{10}$

\section{Conclusion}

This study explored the constructs and interdependency between objectively measured 24-hour physical behaviors and investigated their associations with participant characteristics for people living with COPD (mild-moderate airflow obstruction) and healthy control subjects. Distinct behavioral constructs were identified as "low-intensity movement" (comprising of ST, light activity, and average movement intensity), "high-intensity movement" (comprising of moderate and vigorous activities), and sleep, each independently associated with different participant characteristics. Therefore, being sedentary and being physically active are not two sides of the same coin. Future research should routinely capture, report, and assess the full range of movement intensities (sleep, sedentary, light, moderate, and vigorous) where possible to determine whether modifying these behaviors improves health outcomes in COPD.

\section{Data sharing statement}

The PhARaoH data have been described and made available indefinitely, on request. For conditions of use, please visit the following web link: http://www.lboro.ac.uk/research/ mi-lab/research/pharaoh/pharaohconditionsofuse/. To make an enquiry about using this dataset, please submit a request by visiting the following web link: http://www.lboro.ac.uk/ research/mi-lab/research/pharaoh/. Study documentation, including informed consent form, participant information sheet, and monitor instruction sheet are available on request.

\section{Acknowledgments}

The study was funded by facilitation funds from the NHS England. The authors acknowledge support from the National Institute for Health Research (NIHR) Leicester Biomedical Research Center, which is a partnership between University Hospitals of Leicester NHS Trust, Loughborough University, and the University of Leicester and acknowledge support from the NIHR Collaboration for Leadership in Applied Health Research and Care - East Midlands and The Primary Care Research Network (PCRN). The views expressed are those of the authors and not necessarily those of the NHS, the NIHR, Loughborough University, or the Department of Health.

\section{Author contributions}

MWO had full access to all the data in the study and takes responsibility for the integrity of the data and the accuracy of the data analysis. MWO, MCS, MDM, DWE, APK, LBS, 
and SJS contributed substantially to the study design, data analysis and interpretation; took part in drafting the article or revising it critically for important intellectual content; gave final approval of the version to be published; and agree to be accountable for all aspects of the work.

\section{Disclosure}

The authors report no conflicts of interest in this work.

\section{References}

1. Gimeno-Santos E, Frei A, Steurer-Stey C, et al. Determinants and outcomes of physical activity in patients with COPD: a systematic review. Thorax. 2014;69(8):731-739.

2. Shrikrishna D, Patel M, Tanner RJ, et al. Quadriceps wasting and physical inactivity in patients with COPD. Eur Respir J. 2012;40(5):1115-1122.

3. van HelvoortHA, Willems LM, Dekhuijzen PR, van Hees HW, Heijdra YF Respiratory constraints during activities in daily life and the impact on health status in patients with early-stage COPD: a cross-sectional study. NPJ Prim Care Respir Med. 2016;26:16054.

4. Garcia-Aymerich J, Lange P, Benet M, Schnohr P, Antó JM. Regular physical activity modifies smoking-related lung function decline and reduces risk of chronic obstructive pulmonary disease: a populationbased cohort study. Am J Respir Crit Care Med. 2007;175(5):458-463.

5. Vogelmeier CF, Criner GJ, Martinez FJ, et al. Global strategy for the diagnosis, management and prevention of chronic obstructive lung disease 2017 report: gold executive summary. Respirology. 2017;22(3):575-601.

6. Byrom B, Rowe DA. Measuring free-living physical activity in COPD patients: deriving methodology standards for clinical trials through a review of research studies. Contemp Clin Trials. 2016;47:172-184.

7. Donaire-Gonzalez D, Gimeno-Santos E, Balcells E, et al. Benefits of physical activity on COPD hospitalisation depend on intensity. Eur Respir J. 2015;46(5):1281-1289.

8. Pitta F, Troosters T, Spruit MA, Probst VS, Decramer M, Gosselink R. Characteristics of physical activities in daily life in chronic obstructive pulmonary disease. Am J Respir Crit Care Med. 2005;171(9):972-977.

9. Park SK, Richardson CR, Holleman RG, Larson JL. Physical activity in people with COPD, using the National Health and nutrition evaluation survey dataset (2003-2006). Heart Lung. 2013;42(4):235-240.

10. Tremblay MS, Aubert S, Barnes JD, et al. Sedentary Behavior Research Network (SBRN) - Terminology Consensus Project process and outcome. Int J Behav Nutr Phys Act. 2017;14(1):75.

11. Hill K, Gardiner PA, Cavalheri V, Jenkins SC, Healy GN. Physical activity and sedentary behaviour: applying lessons to chronic obstructive pulmonary disease. Intern Med J. 2015;45(5):474-482.

12. Singh S. Physical activity and pulmonary rehabilitation - A competing agenda? Chron Respir Dis. 2014;11(4):187-189.

13. Orme MW, Esliger DW, Kingsnorth AP, et al. Physical activity and respiratory health (Pharaoh): data from a cross-sectional study. Open Health Data. 2016;4(1):4.

14. Williams ED, Stamatakis E, Chandola T, Hamer M. Assessment of physical activity levels in South Asians in the UK: findings from the health survey for England. J Epidemiol Community Health. 2011;65(6):517-521.

15. Troiano RP, Berrigan D, Dodd KW, Mâsse LC, Tilert T, Mcdowell M Physical activity in the United States measured by accelerometer. Med Sci Sports Exerc. 2008;40(1):181-188.

16. Choi L, Liu Z, Matthews CE, Buchowski MS. Validation of accelerometer wear and nonwear time classification algorithm. Med Sci Sports Exerc. 2011;43(2):357-364.

17. Choi L, Ward SC, Schnelle JF, Buchowski MS. Assessment of wear/ nonwear time classification algorithms for triaxial accelerometer. Med Sci Sports Exerc. 2012;44(10):2009-2016.

18. Kamada M, Shiroma EJ, Harris TB, Lee IM. Comparison of physical activity assessed using hip- and wrist-worn accelerometers. Gait Posture. 2016;44:23-28.
19. Carney CE, Lajos LE, Waters WF. Wrist actigraph versus self-report in normal sleepers: sleep schedule adherence and self-report validity. Behav Sleep Med. 2004;2(3):134-143.

20. WHO. Obesity: Preventing and Managing the Global Epidemic. Geneva: World Health Organisation; 2000.

21. Miller MR, Hankinson J, Brusasco V. Standardisation of spirometry. Series "ATS/ERS task force: standardisation of lung function testing." Eur Respir J. 2005;26:319-338.

22. Pauwels RA, Buist AS, Calverley PMA, Jenkins CR, Hurd SS Global strategy for the diagnosis, management, and prevention of chronic obstructive pulmonary disease. Am J Respir Crit Care Med. 2001;163(5):1256-1276.

23. Quanjer PH, Tammeling GJ, Cotes JE, Pedersen OF, Peslin R, Yernault JC. Lung volumes and forced ventilatory flows. Eur Respir J. 1993;6(Suppl 16):5-40.

24. Singh SJ, Morgan MD, Scott S, Walters D, Hardman AE. Development of a shuttle walking test of disability in patients with chronic airways obstruction. Thorax. 1992;47(12):1019-1024.

25. Edwards RHT, Young A, Hosking GP, Jones DA. Human skeletal muscle function: description of tests and normal values. Clinical Science. 1977;52(3):283-290.

26. Parvatikar VB, Mukkannavar PB. Comparative study of grip strength in different positions of shoulder and elbow with wrist in neutral and extension positions. J Exerc Sci Physiother. 2009;5(2):67.

27. Bestall JC, Paul EA, Garrod R, Garnham R, Jones PW, Wedzicha JA Usefulness of the medical Research Council (MRC) Dyspnoea Scale as a measure of disability in patients with chronic obstructive pulmonary disease. Thorax. 1999;54(7):581-586.

28. Herdman M, Gudex C, Lloyd A, et al. Development and preliminary testing of the new five-level version of EQ-5D (EQ-5D-5L). Qual Life Res. 2011;20(10):1727-1736.

29. van Hout B, Janssen MF, Feng YS, et al. Interim scoring for the EQ5D-5L: mapping the EQ-5D-5L to EQ-5D-3L value sets. Value Health. 2012;15(5):708-715.

30. Lacasse Y, Goldstein R, Lasserson TJ, Martin S. Pulmonary rehabilitation for chronic obstructive pulmonary disease. Cochrane Database Syst Rev. 2006;4:CD003793.

31. Mesquita R, Meijer K, Pitta F, et al. Changes in physical activity and sedentary behaviour following pulmonary rehabilitation in patients with COPD. Respir Med. 2017;126:122-129.

32. Chastin SF, Palarea-Albaladejo J, Dontje ML, Skelton DA. Combined effects of time spent in physical activity, sedentary behaviors and sleep on obesity and Cardio-Metabolic health markers: a novel compositional data analysis approach. PLoS One. 2015;10(10):e0139984.

33. Mesquita R, Spina G, Pitta F, et al. Physical activity patterns and clusters in 1001 patients with COPD. Chron Respir Dis. 2017;14(3): 256-269.

34. Spina G, Spruit MA, Alison J, et al. Analysis of nocturnal actigraphic sleep measures in patients with COPD and their association with daytime physical activity. Thorax. 2017;72(8):694-701.

35. Jácome C, Marques A. Short- and long-term effects of pulmonary rehabilitation in patients with mild COPD: a comparison with patients with moderate to severe COPD. J Cardiopulm Rehabil Prev. 2016;36(6):445-453.

36. Steiner M, Holzhauer-Barrie J, Lowe D, et al. Pulmonary Rehabilitation: Time to breath better. National Chronic Obstructive Pulmonary Disease (COPD) Audit Programme: Resources and organisation of Pulmonary Rehabilitation services in England and Wales 2015. 2015. Available from: https://www.rcplondon.ac.uk/projects/outputs/pulmonaryrehabilitation-time-breathe-better. Accessed February 2, 2019.

37. Egan C, Deering BM, Blake C, et al. Short term and long term effects of pulmonary rehabilitation on physical activity in COPD. Respir Med. 2012;106(12):1671-1679.

38. Watson D. Improving Health in Leicester, Annual Report of the Director of Public Health and Health Improvement 2010. Leicester: NHS Leicester City; 2010. Available from: http://www.cabinet.leicester. gov.uk/documents/s26416/DPH\%20Annual\%20Report\%202010\%20 High\%20Res.pdf. Accessed January 21, 2019. 


\section{Supplementary materials Reliability testing}

Accelerometers are commonplace in the field of $\mathrm{PA}$ research, in part due to their small size, light weight, ability to measure human movement (acceleration), and store data over many days. ${ }^{1}$ Despite the capability of these devices to quantify acceleration with high sensitivity (eg, acceleration data can be recorded 100 times every second; $100 \mathrm{~Hz}$ ) it is a good practice to check that accelerometers are working within an acceptable measurement error before deployment. This is particularly important when large number of devices are being deployed in a single study due to the increased likelihood of inter-device variability. There are plethora of examples of studies examining the validity of accelerometers in both controlled and free-living conditions using human participants. ${ }^{2,3,11,12}$ However, variations in the participants themselves even when a single person wears multiple devices, ${ }^{3}$ introduce inherent variability in the assessment of monitor accuracy. An alternative approach for examining the accuracy of accelerometers has been through the use of mechanical shakers. ${ }^{4,5}$ The advantages of using shakers include the large number of accelerations that can be produced, the ability to assess many accelerometers at once, and the reliable and precise oscillations that can be produced. ${ }^{1}$ The importance of limiting inter-device variation and using devices with acceptable measurement errors is pivotal for accurate and reliable behavior quantification as greater magnitudes of acceleration occur at the wrist compared to locations closer to the center of mass. ${ }^{6}$

\section{Mechanical shaking and inter-device variability}

One hundred and fifty-five ActiGraph wGT3x-BT accelerometers (ActiGraph, LLC) were assessed using an orbital shaker table to provide the researcher full control of the magnitude of the acceleration and the frequency of the oscillation the devices were exposed to. Five different conditions were selected to produce a range of physiologically relevant accelerometer counts within the confines of the shaker capacity; these were 100, 125, 150, 175, and 200 revolutions per minute. Each condition was time-stamped and lasted 2.5 minutes with 1.25 minutes between each condition to allow time for the orbital shaker to adjust the oscillation frequency. Care was taken to secure the monitors, being firmly fixed in a vertical position along their sensitive axis in order to maximize and standardize the output. Once all accelerometers were in position the orbital shaker was switched on and allowed to warm up in order to facilitate the optimal execution of the conditions.
In order to identify accelerometers working outside acceptable limits ie, $\pm 10 \%$ as per manufacturer guidelines, the mean difference percent (Equation S1) was calculated for each unit and visualized using Bland-Altman plots for each condition. Units which exceeded this tolerance were deemed "out of calibration" and returned to the manufacturer. Twelve accelerometers $(7.7 \%)$ were returned to the manufacturer and 113 devices $(72.9 \%)$ were used as part of the study.

$$
\begin{aligned}
& \text { Mean difference percent }= \\
& \frac{\text { Unit specific mean }- \text { Condition grand mean }}{\text { Condition grand mean }} \times 100
\end{aligned}
$$

\section{Data processing}

Wrist-worn accelerometry is in its infancy within the field of PA and sedentary behavior measurement, but there is general consensus and initial evidence to suggest that this location will permit improvements in wear time compliance, ${ }^{7}$ an advantage for capturing data representative of the wearer's usual activities both within and between days. The main reason for this is likely the added comfort for the participant, which enables them to wear the device without disturbing sleep. As a result, participants were only asked to take off the monitor for waterbased activities such as showering. However, with this comes the challenge of differentiating time in waking and non-waking behaviors. Traditional approaches have utilized participant diaries, whereby individuals record the time they went to bed and the time they got up each day, but this is plagued by recall inaccuracies and adds to the burden of study participation. Data-driven approaches are needed to objectively identify sleep onset and end without the additional burden to participants.

\section{Location and device setup}

Objectively derived PA and sedentary time were collected using the ActiGraph wGT3X-BT accelerometer worn on the nondominant wrist (non-writing hand) continuously except for water-based activities at a sample rate of $100 \mathrm{~Hz}$. Monitors were deployed in delay mode on day 0 and commenced logging on day 1 at 00:00 with a 7-day stop time indicated. Each accelerometer was returned via mail after seven full days of wear. Monitors were initialized and downloaded using ActiLife software (ActiGraph, LLC) version 6.13.2 and were analyzed using KineSoft (KineSoft, Loughborough, UK) version 3.3.80. Data were processed in 60-second epochs.

\section{Preprocessing accelerometry analysis}

Sixty-second, agd files were processed through KineSoft using Choi wear-time criteria ${ }^{8,9}$ to identify periods of 
non-wear. Individual files were exported in "processed mode" using the File Inspector function in KineSoft. The processed data (ie, with non-wear coded) were then inserted into an automated sleep detection system.

\section{Identifying time in bed and out of bed}

Sleep detection was determined using sustained periods of (in)activity from vector magnitude count values based on the work of Carney et al. ${ }^{10}$ To identify the time when participants went to sleep (INBED), the algorithm identified consecutive dips in activity, specifically a $90 \%$ reduction from the previous epoch for 15 minutes between the hours of 21:00 and 23:59. ${ }^{10}$ Once the INBED criteria were met, the original epoch containing the $90 \%$ reduction in counts was used to signify the start of sleep. To identify the time when participants were awake (OUTBED), the algorithm detected consecutive rises in activity level of at least $75 \%$ from the previous epoch for 5 minutes between 06:00 and 09:00. ${ }^{10}$ Once the OUTBED criteria were met, the original epoch containing the $75 \%$ increase in counts was used to signify the end of sleep.

\section{Window identification}

In order to facilitate the aforementioned algorithm, a subsample of 80 files (comprising 20 apparently healthy males, 20 apparently healthy females, 20 male COPD patients, and 20 female COPD patients) was used to visually inspect the suitability of using the 06:00-09:00 and 21:00-23:59 windows as part of the sleep detection verification process. Minute-by-minute vector magnitude was plotted for each of the 7 days of wear and subjected to visual inspection for spikes in activity between 06:00 and 09:00 and dips in activity between 21:00 and 00:00. Whilst these patterns were consistently observed, for the 06:00-09:00 window it was noticed that the activity was still relatively high before this window for some individuals, therefore, additional criteria were included to identify OUTBED occurrences prior to 06:00 and after 09:00. Similarly, between 21:00 and 00:00 it was noticed that the activity was still relatively high after this window for some individuals, therefore, additional criteria were included to identify INBED occurrences after 00:00. Consequently, additional criteria were put in place to account for variation in sleep/wake cycles between participants.

\section{Postprocessing data checking}

If no INBED occurrence from 21:00 to 23:59 was identified, the algorithm used a default time stamp of 23:59 and the file was flagged for visual inspection to determine the exact INBED occurrence. For INBED time stamps that complied with the 21:00-23:59 window, visual inspection was required if additional time stamps were present. The default time stamp (first occurrence) was altered based on visual inspection if subsequent spikes in activity lasted at least 2 minutes at light or moderate intensity, or 5 minutes if sedentary intensity was present. For all OUTBED time stamps, an automated time-stamped detection of sustained spikes in vector magnitude was conducted. Files were flagged for visual inspection if a spike in activity occurred within 1 hour of the algorithm-derived time stamp.

\section{Accelerometry algorithm alterations}

Of the 436 total accelerometry files, 435 (99.8\%) files were visually inspected for at least 1 day for either INBED or OUTBED classification. The whole sample of 436 files provided a total number of 3,052 potential days of wear for the PhARaoH participants. Of these, 2,437 (79.8\%) required visual inspection for INBED detection of which $1,515(62.2 \%)$ required an alteration to the algorithm time stamp (originally windowed between 21:00 and 23:59). For OUTBED detection, 694 (22.7\%) days required visual inspection of which $598(86.0 \%)$ required an alteration to the algorithm time stamp (originally windowed after 06:00).

For the 1,515 INBED detections requiring alterations from the original algorithm-derived time stamps, 596 (39.3\%) were due to participants going to sleep after midnight, $870(57.4 \%)$ were from adjustments made to the first time stamp after $21: 00$, and $49(3.2 \%)$ were from visual inspection alone. Of the remaining 922 days the algorithm was not altered with $203(22.0 \%)$ because periods of non-wear were detected, $63(6.8 \%)$ were for day 7 defaulting to 23:59, and $10(1.1 \%)$ were not altered following visual inspection.

For the 598 OUTBED detections requiring amendment from the original algorithm output, $540(90.3 \%)$ were due to participants waking up before $06: 00$ and 58 (9.7\%) were from visual inspection alone. Of the remaining 96 days the algorithm was not altered with 57 (59.4\%) due to periods of non-wear and 39 (40.6\%) were not altered following visual inspection.

\section{Accelerometry processing}

After establishing the INBED and OUTBED times for each day, sleep was coded as 0 counts (equivalent to non-wear) in order to be removed by non-wear algorithm during data processing. As a result, time spent in activity intensities was derived from waking wear time. Time spent sleeping was calculated from the time between INBED and OUTBED occurrences. VMCPM was calculated by dividing average total counts per day by average waking wear time. 


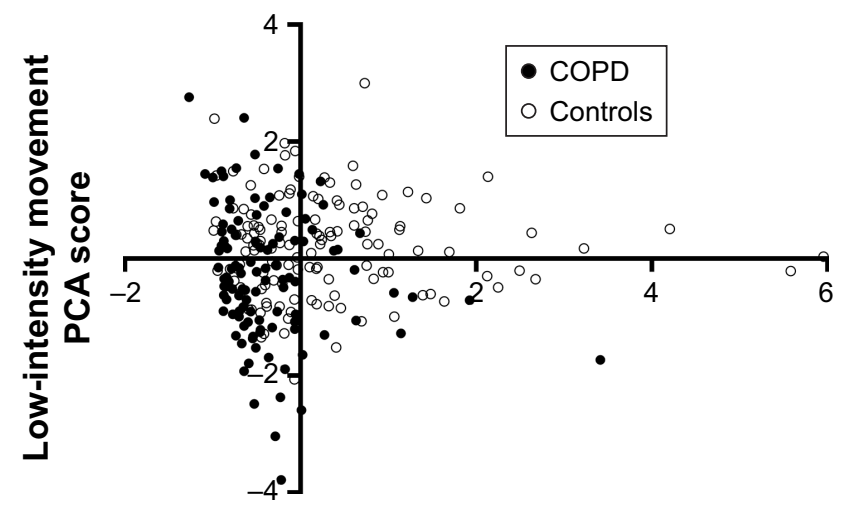

\section{High-intensity movement \\ PCA score}

Figure SI Scatterplot of component scores for "low-intensity movement" and "high-intensity movement."

Notes: Solid circles: COPD; blank circles: controls.

Abbreviation: PCA, principal components analysis.

PA intensities were defined according to published cut points for sedentary time, light intensity activity, and MVPA. ST was defined as $<2,000$ VMCPM, LPA as 2,000-7,499 VMCPM, MVPA as $\geq 7,500 \mathrm{VMCPM}$, and VPA as $\geq 8,250$ VMCPM. ${ }^{6}$

\section{References}

1. Rothney MP, Apker GA, Song Y, Chen KY. Comparing the performance of three generations of ActiGraph accelerometers. J Appl Physiol. (1985) 2008;105(4):1091-1097.

2. Welk GJ. Principles of design and analyses for the calibration of accelerometry-based activity monitors. Med Sci Sports Exerc. 2005; 37(11 Suppl):S501-S511.

3. Nichols JF, Morgan CG, Chabot LE, Sallis JF, Calfas KJ. Assessment of physical activity with the Computer Science and Applications, Inc., accelerometer: laboratory versus field validation. Res $Q$ Exerc Sport. 2000;71(1):36-43.

4. Metcalf BS, Curnow JS, Evans C, Voss LD, Wilkin TJ. Technical reliability of the CSA activity monitor: The Early Bird Study. Med Sci Sports Exerc. 2002;34(9):1533-1537.

5. Esliger DW, Tremblay MS. Technical reliability assessment of three accelerometer models in a mechanical setup. Med Sci Sports Exerc. 2006;38(12):2173-2181.
6. Kamada M, Shiroma EJ, Harris TB, Lee I. Comparison of physical activity assessed using hip- and waist-worn accelerometers. Gait Posture. 2016;44:23-28.

7. van Hees VT, Renström F, Wright A, et al. Estimation of daily energy expenditure in pregnant and non-pregnant women using a wrist-worn tri-axial accelerometer. PLoS One. 2011;6(7):e22922.

8. Choi L, Liu Z, Matthews CE, Buchowski MS. Validation of accelerometer wear and nonwear time classification algorithm. Med Sci Sports Exerc. 2011;43:357-364.

9. Choi L, Ward SC, Schnelle JF, Buchowski MS. Assessment of wear/ nonwear time classification algorithms for triaxial accelerometer. Med Sci Sports Exerc. 2012;44:2009-2016.

10. Carney CE, Lajos LE, Waters WF. Wrist ActiGraph versus self-report in normal sleepers: sleep schedule adherence and self-report validity. Behav Sleep Med. 2004;2(3):134-143.

11. Esliger DW, Rowlands AV, Hurst TL, Catt M, Murray P, Eston RG. Validation of the GENEA Accelerometer. Med Sci Sports Exerc. 2011;43(6):1085-1093.

12. Hendelman D, Miller K, Baggett C, Debold E, Freedson P. Validity of accelerometry for the assessment of moderate intensity physical activity in the field. Med Sci Sports Exerc. 2000;32(9 Suppl):S442-S449.

\section{Publish your work in this journal}

The International Journal of COPD is an international, peer-reviewed journal of therapeutics and pharmacology focusing on concise rapid reporting of clinical studies and reviews in COPD. Special focus is given to the pathophysiological processes underlying the disease, intervention programs, patient focused education, and self management protocols.

\section{Dovepress}

This journal is indexed on PubMed Central, MedLine and CAS. The manuscript management system is completely online and includes a very quick and fair peer-review system, which is all easy to use. Visit $\mathrm{http}: / /$ www.dovepress.com/testimonials.php to read real quotes from published authors. 